With regard to Dr. Ascoli's question of the effect of spinal shock on the bladder, this is a subject which will be discussed next year in detail. As you know after spinal cord transection there is only hyoptonia because the autonomous innervation within the bladder wall is still active and some effector response to afferent stimulation (distension) is possible. The bladder will only become atonic if it is allowed to overdistend-then you knock out the peripheral innervation. The overdistension of the bladder musculature produces an additional local effect to the effects produced already by the spinal cord transection, exactly as this occurs in the skeletal muscles. This applies also, of course, to the colon. In cervical cord lesions the immediate effect of cord transection is paralysis of the peristalsis resulting in intestinal distension due to meteorism which in turn has an adverse effect on the function of the diaphragm and thus on the impaired respiration, due to the paralysis of the intercostals. Dr. Silver and I have made extensive electro-myographic studies on the effect of spinal shock on the intercostal muscles published in Paraplegia in 1965. They participate, exactly as any other muscles in the reflex depression during spinal shock, but they also recover reflexly, and in due course, by a co-ordinated reflex function can help in the restoration of the vital capacity of the lung.

Finally, the very important question was asked whether an immediate surgical procedure in a traumatic paraplegia can aid in resolving spinal shock. You heard some laughter amongst the audience when this question was raised: the answer is NO! On the contrary, it just adds to the shock.

\title{
PROFFERED PAPERS
}

\author{
Chairman; Dr. T. GREGG
}

\section{IN DEFENCE OF THE FOSTER-STRYKER TURNING BED}

\author{
By Professor R. Ascoli \\ Milan, Italy
}

I DON'T know the name of the surgeon who said that practically all operating methods are equally satisfactory provided that they are used by an operator who is sufficiently well acquainted with them. I don't interpret this maxim literally, but it is true that there are surgeons and physicians who get excellent results with methods that others would describe as bad. And vice-versa. I would like today to say a word in defence of the turning beds of the Foster-Stryker type in the treatment of paraplegics. I am led to do this by the fact that today in the treatment of paraplegics and of bedsores there is a tendency to abandon the use of these beds for other methods of treatment, which in my view are far less reliable.

We are all acquainted with the principle of these turning beds: the purpose is to turn the bed about a longitudinal axis in such a way that the patient rotates en bloc with the plane on which he is lying, and in such a way that the slightest movement of the body, and in particular of the vertebral column, is impossible.

The chief indication of these beds are vertebral fractures with or without spinal cord lesions where it is of prime importance that the parts of the fracture remain completely immobile, and where it is also essential at intervals to change that part of the body-and in particular the skin-which supports the weight of the body. This aspect is clearly of great importance in the prevention and cure of bedsores: it affects in particular the sacral region, since here the skin is practically in contact with the bone surface underneath, and is especially exposed to ischemic and necrotic processes. 
I would once more emphasise that it is a fundamental requirement that during the change from dorsal to ventral position and vice-versa, there must not be the slightest movement of the fractured spine. Clearly such a necessity does not arise in the case of patients who do not require absolute immobility of the column, for instance non-traumatic paraplegics or even non-paraplegic patients suffering from bedsores.

Now during the manual lifting of the patient by two or three nurses, even if they are highly skilled, there can never be an absolute guarantee that the fractured parts of the spine will not rub one against the other.

Even a femur or tibia fracture may be treated without immobilisation in plaster, provided the patient remains immobile, always in the same position, but if we want to shift him or turn him we cannot avoid some displacement of the fracture stumps. It was just this circumstance that gave rise to the use of plaster apparatus and immobilisation apparatus in general.

Apart from this, the need for periodically changing from the supine to the prone position and vice-versa, and also for permitting a permanent prone position is imperative, for the purpose of preventing dorsal and above all sacral bedsores: it is well known that the latter occur particularly often in paraplegics.

In view of these requirements the Stryker-Foster type of bed was inventedand others of a similar type-comprising not only a single plane for the patient to lie on, but two planes, one ventral and one dorsal. When the patient is in a supine position the ventral frame is removed, when he is in a prone position the dorsal frame is removed. The whole apparatus can rotate through I80 degrees about a longitudinal axis. To turn the patient from a supine to a prone position (or viceversa), both the frames, dorsal and ventral, are applied to the patient, attached one to the other quite tightly: a rapid rotating movement through I80 degrees is then made, whereupon the upper plane is immediately removed.

But the turning bed also presents other advantages. The frames, which are made of strong canvas, do not comprise only one piece, but various segments, and it is possible to create a gap corresponding to the sacral region, so that this region does not rest on any frame, but is, so to speak, suspended in mid-air and this is clearly an immense benefit for the prevention and cure of bedsores. A similar gap in the ventral frame may be obtained, too, at the level of the genital region, avoiding in the case of a male patient a harmful compression on the testicles and in order to permit, when necessary, the passage of vesical drainage.

Moreover the bed is equipped with a device by means of which it is possible to produce some degree of lordosis that may be exploited therapeutically.

In spite of all these advantages there are some spinal cord specialists who are against the use of the turning bed. What are the arguments they put forward?

These authors maintain that it is difficult for the majority of paraplegics to tolerate this turning bed, especially during the first stage, because it is hard and uncomfortable, and in particular these authors state that the prone position is not tolerated.

To these objections I would reply that the greater hardness and rigidity of the bed may be almost entirely neutralised by means of layers of foam rubber, cut to size and applied in the appropriate places. In particular, in the places where parts of the body might come into contact with the longitudinal metal bars of the frame, it is indispensable to apply a layer of foam rubber, which will serve to avoid contact with the hard foreign body. This applies particularly to the iliac regions. 
These are details that are often neglected by those who are not sufficiently well acquainted with the turning bed, and then, of course, results are not good.

The objection that the prone position is often not tolerated by patients, especially during the first stage, is practically speaking unfounded. Because it is by no means necessary that during the first stage, immediately after the trauma, the patient be immediately subjected to a treatment of alternate rotation.

On the contrary, the patient can perfectly well be kept for days on end in a purely supine position, provided that the dangerous area par excellence, the sacral region, coincides with the gap in the dorsal plane in such a way that all pressure is eliminated. And in this connexion I would point out that since not all patients are of the same height, the gap must be adapted according to requirements so that it corresponds precisely with the sacral region. Later on it will be possible to persuade the patient to accept the prone position, starting with a few minutes every day, and increasing the amount of time he spends in that position by a few minutes each day. Recently some electrically-operated beds have been put on the market, such as the Egerton or Egerton Stoke Mandeville tilting and turning beds, which are equipped to turn the bed on which the patient is lying from the supine position to the oblique-lateral/right or to the oblique-lateral/left positions. Apart from the objection of high cost, with these beds it is not possible to achieve an entirely prone position-the position best designed to abolish pressure on the sacral tissues; moreover, we do not think that with these beds the possibility of small movements of fracture stumps is entirely eliminated.

\section{RESULTS}

Our experience of over 12 years' work in the Paraplegic Department of the Milan Traumatologic Center has permitted us to arrive at the following conclusions:

(I) For traumatic paraplegics of very recent date the Foster-Stryker turning bed had proved to be most useful in preventing the formation of bedsores. Of a total of many hundreds of paraplegics treated, only in two or three cases was it impossible to avoid slight decubitus ulcers: this was almost always due to carelessness on the part of nursing staff.

(2) The turning bed is extremely useful in eliminating all possibility of movement by vertebral stumps one against the other.

(3) For paraplegics of not very recent date who at the time of hospitalisation already present sacral decubitus ulcers, the use of the turning bed is invaluable in eliminating or reducing to a minimum the pressure on the region of the decubitus ulcer, and permitting periodical dressing and a rapid cure.

(4) In my view the use of rotations and a periodical change in position also presents the great advantage of keeping the contents of the bladder and also of the renal pelvis in frequent movement: in this way, if any sedimentations of mucus, pus or minerals from at the base of the bladder or pelvis, these, during the jolting due to the change of position, are stimulated to spread once more into the vesical liquid and enter the outlet represented by the catheter (or ureter). In this manner it is far less likely that urinary stones will form. We all know that the main cause of these, in addition to alkaline reaction and the presence of fibrin, blood or pus, is the absolute immobility of the contents of the bladder.

(5) The turning bed, provided it is used with care and adjusted to each particular case, has never been the cause of difficulties of a general nature (serious 
malaise, circulation troubles, respiratory troubles, etc.) or of a local nature (formation of decubitus ulcers in the iliac regions, etc.).

\section{Discussion}

Chairman. One of the little points which we have come to recognise is that when the patient is in the prone position their legs tend to hyper-extend at the knees, and often if the nurses do not adjust the canvas properly there is a foot drop, which may add to the problems when the patient starts to ambulate later on. In fact, I recall several cases where the knees have developed an acute synovitis, which I am sure was simply due to the hyperextension for a:long period of time in the prone position.

J. S. Young (U.S.A.). I join with Professor Ascoli in supporting the use of the Stryker frame. We have used this at Dr Craig's place at Denver for some I4 years: we had three pressure sores develop in I 4 years and they were also of our fault. Some points I would like to make, though, that I think are important. I think the shortness of length of stay that we experienced came a lot from use of the Stryker bed, because we could mobilise these people and get them out of the rooms all over the place, eating in the dining-room, out in the yard, into the therapies-literally all over the place from the onset. I think we developed cardiovascular pulmonary reserve which allowed us to get them up in the upright position within three or four days having been immobilised for six weeks. I think this vasomotor conditioning a part of the Stryker frame. Also, it is very adaptable to, for example, ball-bearing features. With the tetraplegic, he lies on the frame and he literally works his arms by the hour, he doesn't have to have immediate supervision by the occupational therapist or physical therapist. I think this is an excellent device and I don't think it should be abandoned in favour of all our mechanical equipment which somehow seems to be attractive but is rather unphysiological.

P. HARRIS (G.B.). Some years ago at this meeting we read a paper on these frames stressing the point in conjunction with Blackburn's skull calipers, and one of the advantages of using this type of frame would appear to be the use of the caliper and providing suitable fixation of the cervical spine. There are some problems with these frames which the speaker might care to enlarge on: the problem of stasis in the leg veins, which we have overcome in a simple way, but we would like to hear him on this, possibly. We do not use any foam plastic mattress, we prefer pillows, soft pillows being better, and also in relation to the use of these frames and in regard to what our President said this morning about the position of the patient, and in discouraging flexion spasms and contractures, we think that the prone position for paraplegics and tetraplegics is an important position, from an early stage after they have sustained their lesion.

L. S. Michaelis (G.B.). We knew, of course, the Stryker-Foster frames, we were not fond of the prone position, particularly for those patients who, in addition to their spinal injury, also had severe thoracic injuries, fractures of the ribs, haemothorax or pneumothorax. In addition, we were not very happy about the position of the arms in the tetraplegics-it seemed to us that these frames were too narrow. That is why we first of all eliminated the need for lying prone on this particular bed-we have other means of changing patient's position-by providing the lateral turning bed, and I believe that certainly for those people with associated injuries of the chest and for the cervical patient the Stoke Mandeville-Egerton bed has something which the Stryker frame cannot provide.

Sir Ludwig Guttmann (G.B.). It is quite true that tetraplegics can be treated in prone position, but they should not be continuously in prone position as otherwise they 
will develop contractures in extension. These people are left lying, sometimes for weeks, with extended legs. Only three months ago I saw a tetraplegic girl in an orthopaedic unit in prone position on a Foster frame, and when I tried to move her legs, they were completely stiff and the X-ray showed already peri-articular changes. The disadvantages of the Stryker-Foster frame is that there are only two possibilities-supine and prone position which is not satisfactory. For many years we have done research on turning beds to change from the supine to lateral position, which eventually has proved, I think, to be highly successful. There are, of course, modifications of this bed, and in Dr. Gregg's Unit there is a similar turning bed, constructed on the principle of the Stoke Mandeville bed but provided with a motor which turns the patient continuously slowly. Well, of course, one can do the lateral turning with the Stoke Mandeville bed by pressing a button, the only difference is that the other bed will be more expensive.

\section{T. GREGG (Ireland). Well, there are other differences.}

Sir Ludwig Guttmann (G.B.). Anyway, I think I must warn you to avoid both the permanent extension and flexion, otherwise contractures will develop, and that is just what you don't want. There is another point worth while mentioning with regard to the disadvantage of the Stryker-Foster bed. I saw in the United States, for instance, tetraplegics after lying flat in pronation with the normal contour of the spine completely changed and flattened out. This is a serious disadvantage of the Stryker frame. On the other hand to keep paraplegics and tetraplegics in the acute stages 'days on end' in supine position is certainly not correct.

Professor Ascoli (Italy). My purpose was only to communicate our results and I may say they are very good. Naturally, you must take care of these patients, and when you turn them you must see that everything is in order, and in each case you must decide the way you should treat him. Of course, in the beginning it is impossible to leave the patient prone for a long time they don't tolerate this. But it is quite possible to have those patients days and days lying in supine position without turning them provided that the sacral region-the most vulnerable region for bedsores-lies between the widest space of the gap that fixes this bed. Tomorrow you will see in a film that we have now an Italian made bed, which you can have larger and narrower. This is very important, I think, in reply to Dr. Michaelis who said that the bed is too narrow. With a particular screw mechanism-there are four screws-you can enlarge the bed or make it narrower. This is very important to adapt it to each patient. When patients come to us without bedsores they never get bedsores. I must add another thing: if I have understood Sir Ludwig correctly, he said that this bed places the spine absolutely straight horizontally. In our recent models there is a possibility to angulate the bed to produce a lordosis of the spine.

Chairman. We have had a very good discussion and Professor Ascoli got, on the whole a favourable reception to his paper, with strong backing from North America and Canada. Each of us develops our ideas, which we consider the best. In Dublin, we think it much more physiological to keep the patient moving continuously rather than to be turned occasionally, and not only do we not cause bedsores, but most bedsores, even of 6 inches in diameter, will heal on this bed whilst the patient is in supine position. We thank Professor Ascoli very much for bringing this to us, which has been a question of contention, and he has vindicated the Stryker-type of frame. 